\title{
Nuove applicazioni della medicina nucleare in ambito diagnostico (I parte) ${ }^{\text {th }}$
}

\section{Recent applications of nuclear medicine in diagnostics (I part)}

\section{Giorgio Treglia $^{a, *}$, Ernesto Cason ${ }^{b}$, Giorgio Fagioli ${ }^{b}$}

\author{
"Istituto di Medicina Nucleare, Policlinico "A. Gemelli", Università Cattolica del Sacro Cuore, Roma \\ ' UO Medicina Nucleare, Ospedale "Maggiore", ASL di Bologna
}

Ricevuto il 22 ottobre 2009; accettato il 29 ottobre 2009

disponibile online il 19 marzo 2010

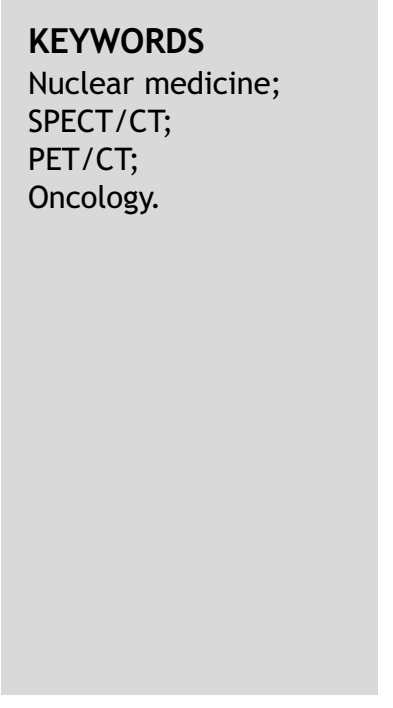

\begin{abstract}
Summary
Introduction: Aim of this review is to describe the recent applications of nuclear medicine techniques in diagnostics, particularly in oncology.

Materials and methods: We reviewed scientific literature data searching for the current role of tomographic nuclear medicine techniques (SPECT and PET) in oncology and summarized the main applications of these techniques.

Results: Nuclear medicine techniques have a key role in oncology allowing early diagnosis of many tumours, an accurate staging of disease and evalutation of treatment response. Hybrid SPECT/CT and PET/CT imaging systems now provide metabolic and functional information from SPECT or PET combined with the high spatial resolution and anatomic information of CT. The most frequent applications of SPECT/CT in oncology concern thyroid tumours, neuroendocrine tumours, bone metastases and lymph node mapping. Furthermore the evaluation of many tumours may benefit from PET/CT imaging.

Discussion: The recent development of new radiopharmaceuticals and the growth of hybrid tomographic devices, such as SPECT/CT and PET/CT, now permits molecular imaging of biologic processes at the cellular level to improve both the diagnosis and treatment of many tumours. (c) 2010 Elsevier Srl. All rights reserved.
\end{abstract}

\footnotetext{
La seconda parte del presente articolo sarà pubblicata sul n. 3/2010 dell'Italian Journal of Medicine.

* Corrispondenza: Istituto di Medicina Nucleare, Policlinico "A. Gemelli”, Università Cattolica del Sacro Cuore, l.go Gemelli 8 - 00168 Roma. E-mail: giorgiomednuc@libero.it (G. Treglia).
} 


\section{Introduzione}

Il presente articolo, suddiviso in due parti, si propone di fornire un aggiornamento sulle metodiche di imaging mediconucleare attualmente in uso, con particolare riguardo ai nuovi sviluppi nel campo dell'Oncologia, della Neurologia, della Cardiologia e dello studio dei processi infiammatori.

Le metodiche di medicina nucleare possono fornire importanti informazioni sul metabolismo corporeo e sulla biologia cellulare, generando immagini funzionali non ottenibili con altre tecniche diagnostiche. Questo ruolo, la cui peculiare importanza è da tempo riconosciuta, deriva dalla possibilità di disporre di traccianti, i radiofarmaci, idonei a definire specifiche vie metaboliche e di apparecchiature, le gammacamere e le camere a positroni, in grado di rilevarne la distribuzione all'interno del corpo e di generare immagini funzionali.

Notevoli progressi sono stati compiuti in anni recenti sia nel campo dei radiofarmaci sia in quello degli apparecchi di rilevazione e attualmente si sono differenziate due aree dell'imaging funzionale medico-nucleare: la cosiddetta "diagnostica convenzionale", che impiega radiofarmaci gammaemittenti (detti anche a singolo fotone) e come apparati di rilevazione le gammacamere in configurazione planare o tomografica SPECT (acronimo dell'inglese Single Photon Emission Computerized Tomography); la "diagnostica PET" (acronimo di Positron Emission Tomography), che utilizza radiofarmaci emittenti positroni e appositi tomografi per la rilevazione della radioattività [1].

I radiofarmaci usati in medicina nucleare per la rilevazione con gammacamere convenzionali contengono isotopi che emettono fotoni gamma (tabella 1).

Il radionuclide più comunemente impiegato è il tecnezio$99 \mathrm{~m}$, la cui energia ( $140 \mathrm{KeV})$ si adatta bene alla rilevazione da parte dei cristalli di ioduro di sodio delle comuni gammacamere e la cui breve emivita (6 ore) consente un ridotto tempo di radioesposizione. Con questo isotopo è possibile marcare una grande varietà di traccianti con tropismo specifico per differenti tessuti, normali e patologici. Un altro radionuclide gamma-emittente è l'indio-111 (picchi di energia a 171 e $245 \mathrm{KeV}$ ed emivita di 68 ore), che può essere legato a peptidi (per esempio, ${ }^{111} \mathrm{In}$-pentetreotide, radiofarmaco utile per la rilevazione dei tumori neuroendocrini). Altro emettitore di singolo fotone è lo iodio-123 che, legato per esempio a composti analoghi dell'adrenalina come la metaiodobenzilguanidina (MIBG), permette di studiare $i$ tumori derivanti dalla cresta neurale e l'innervazione adrenergica cardiaca. Lo iodio-131 (gamma e beta-emittente; emivita di circa 8 giorni) è invece un radioisotopo sfruttato in medicina nucleare a scopo sia diagnostico (per la sua

Tabella 1 Radionuclidi emettitori di singolo fotone.

\begin{tabular}{lll}
\hline Radionuclide & Emivita & Energia $(\mathrm{KeV})$ \\
\hline Tecnezio-99m $\left({ }^{99 \mathrm{~m}} \mathrm{Tc}\right)$ & 6 ore & 140 \\
\hline lodio-131 $\left({ }^{131} \mathrm{I}\right)$ & 8 giorni & 364 \\
\hline lodio-123 $\left({ }^{123} \mathrm{I}\right)$ & 13 ore & 159 \\
\hline Gallio-67 $\left({ }^{67} \mathrm{Ga}\right)$ & 78 ore & $93-296$ \\
\hline Indio-111 $\left({ }^{111} \mathrm{In}\right)$ & 68 ore & $173-247$ \\
\hline
\end{tabular}

Tabella 2 Radionuclidi emettitori di positroni.

\begin{tabular}{llc}
\hline Radionuclide & Emivita & Energia beta + max (MeV) \\
\hline Fluoro-18 $\left({ }^{18} \mathrm{~F}\right)$ & $110 \mathrm{~min}$ & 0,635 \\
\hline Carbonio-11 $\left({ }^{11} \mathrm{C}\right)$ & $20 \mathrm{~min}$ & 0,96 \\
\hline Ossigeno-15 $\left({ }^{15} \mathrm{O}\right)$ & $2 \mathrm{~min}$ & 1,723 \\
\hline Azoto-13 $\left({ }^{13} \mathrm{~N}\right)$ & $10 \mathrm{~min}$ & 1,19 \\
\hline Rame-64 $\left({ }^{64} \mathrm{Cu}\right)$ & $770 \mathrm{~min}$ & 0,654 \\
\hline lodio-124 $\left({ }^{124} \mathrm{I}\right)$ & 4 giorni & 2,13 \\
\hline Gallio-68 $\left({ }^{68} \mathrm{Ga}\right)$ & $68 \mathrm{~min}$ & 1,9 \\
\hline
\end{tabular}

emissione gamma) sia terapeutico (per l'emissione beta) in particolare nello studio dei carcinomi tiroidei differenziati. Il gallio-67 (emivita 78 ore), infine, è utilizzato in medicina nucleare come gallio-citrato per lo studio di processi flogistici e come tracciante oncotropo (tabella 1) [1].

Fin dalla metà degli anni Ottanta in medicina nucleare "convenzionale" è stato possibile effettuare, oltre alle consuete rilevazioni planari bidimensionali, rilevazioni tomografiche SPECT; mentre la SPECT è, allo stato attuale, relativamente meno costosa e più ampiamente disponibile rispetto alla PET, va rilevato che gli apparecchi SPECT hanno una risoluzione spaziale ridotta rispetto ai tomografi PET.

La PET consente di rilevare le radiazioni emesse da radionuclidi emettitori di positroni (tabella 2) legati a vari composti, con lo scopo di studiare differenti processi metabolici.

L'immagine PET viene ottenuta mediante la rilevazione di coppie di fotoni (di $511 \mathrm{KeV}$ ciascuno) che vengono prodotte dall'annichilazione di un positrone con un elettrone. Il radiocomposto più utilizzato in PET, allo stato attuale, è il fluoro18-fluorodesossiglucosio (FDG), analogo del glucosio e pertanto particolarmente utile nello studio del metabolismo glucidico. Altri radiofarmaci PET permettono di studiare diversi processi metabolici quali sintesi proteica, espressione genica, ipossia cellulare e stato recettoriale (tabella 3) [1].

La SPECT e la PET, per la ridotta risoluzione spaziale delle metodiche medico-nucleari, forniscono informazioni

Tabella 3 Radiofarmaci PET: via metabolica indagata.

\begin{tabular}{ll}
\hline Via metabolica & Radiofarmaci \\
\hline Metabolismo glucidico & ${ }^{18} \mathrm{~F}$-fluorodesossiglucosio (FDG) \\
\hline Sintesi proteica & ${ }^{11} \mathrm{C}$ - metionina, ${ }^{18} \mathrm{~F}$-timidina \\
\hline $\begin{array}{l}\text { Trasporto aminoacidico/ } \\
\text { sintesi DNA }\end{array}$ & ${ }^{11} \mathrm{C}$-tirosina, ${ }^{18} \mathrm{~F}$-tirosina, \\
\hline $\begin{array}{l}\text { Metabolismo della } \\
\text { membrana cellulare }\end{array}$ & ${ }^{11} \mathrm{C}$-timidina \\
\hline Ipossia tissutale & ${ }^{11} \mathrm{C}$-acetana, ${ }^{18} \mathrm{~F}$-colina, \\
\hline Stato recettoriale & ${ }^{18} \mathrm{~F}$-misonidazolo \\
\hline Consumo di ossigeno & ${ }^{18} \mathrm{~F}$-beta-estradiolo, \\
\hline Flusso ematico & ${ }^{15} \mathrm{Ga}$-DOTANOC, ${ }^{68} \mathrm{Ga}$-DOTATOC \\
\hline Metabolismo osseo & ${ }^{13} \mathrm{~N}$-ammoniaca \\
\hline Apoptosi & ${ }^{18} \mathrm{~F}$-fluoruro \\
\hline
\end{tabular}


anatomiche inferiori rispetto alle metodiche radiologiche come la tomografia computerizzata (TC) e la risonanza magnetica (RM). Questo problema è stato superato dal recente sviluppo di sistemi diagnostici integrati (SPET/TC e $\mathrm{PET} / \mathrm{TC}$ ) in grado di fondere le informazioni funzionali,

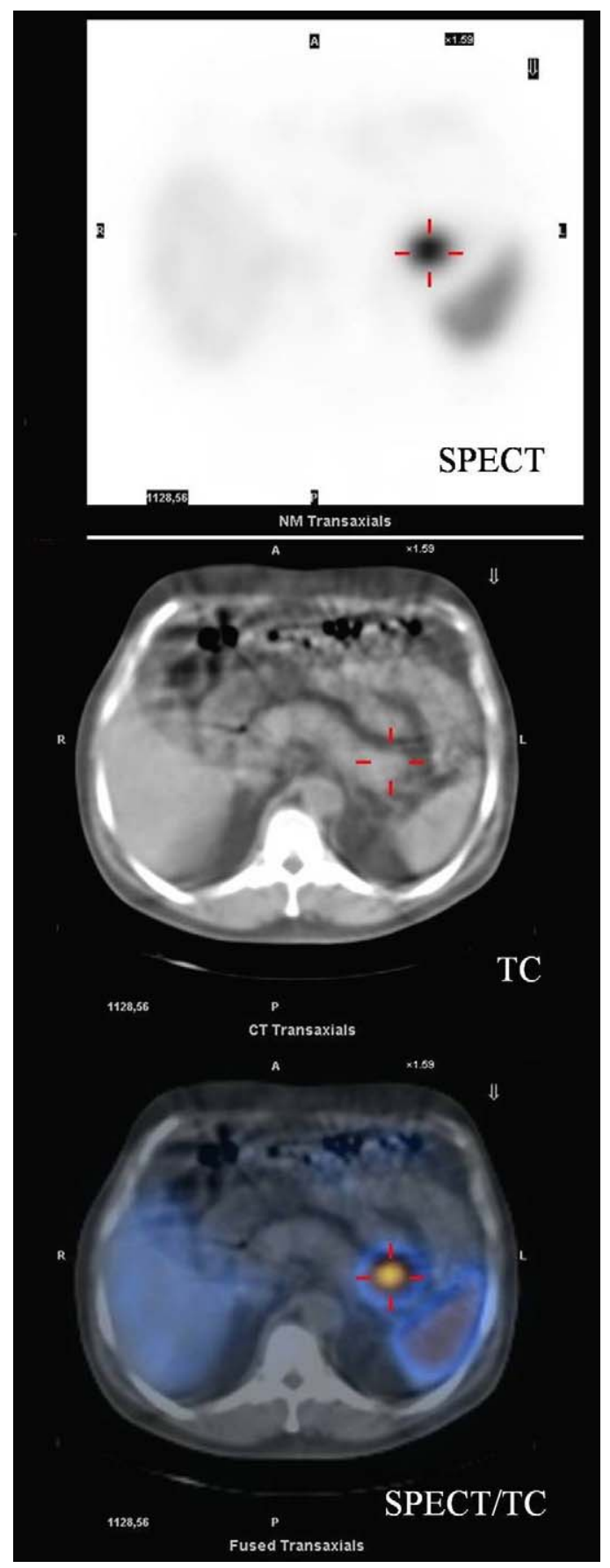

Figura 1 Paziente di 65 anni con lesione della coda pancreatica di riscontro TC, di sospetta natura neuroendocrina. L'indagine scintigrafica con analoghi marcati della somatostatina (indio111-pentetreotide), effettuata con metodica SPECT/TC, ha dimostrato iperaccumulo del radiocomposto in corrispondenza di un'area nodulare a livello della coda pancreatica, documentando pertanto la presenza di tessuto neuroendocrino in tale sede. derivanti dalle metodiche medico-nucleari, con quelle anatomiche, derivanti dalle metodiche radiologiche ( figg. 1 e 2): ciò ha permesso di ottenere maggiori informazioni diagnostiche rispetto alle singole metodiche considerate singolarmente. In particolare, grazie alla fusione dei due gruppi di immagini, funzionali e anatomiche, è possibile attribuire una precisa localizzazione anatomica a un'area di alterato metabolismo. Allo stato attuale la componente TC dell'imaging ibrido è utilizzata soprattutto come TC senza mezzo di contrasto, per la correzione dell'attenuazione e per la localizzazione anatomica; tuttavia, sono in corso alcuni studi per dimostrare i vantaggi dell'uso della PET/TC con mezzo di contrasto rispetto alla PET/TC senza mezzo di contrasto.

I sistemi diagnostici integrati hanno dimostrato il loro valore clinico soprattutto in campo oncologico, ma le applicazioni si sono estese anche in campo cardiologico, neurologico e nell'imaging dei processi infiammatori. Allo stato attuale l'imaging molecolare, definito come la caratterizzazione e la misura dei processi biologici in vivo a livello cellulare e molecolare, ha trovato ampia enfasi grazie specialmente alle informazioni metaboliche fornite dai sistemi ibridi PET/TC [1].

Prospettive future sono rappresentate dallo sviluppo di nuove apparecchiature (per esempio, sistemi ibridi PET/RM) e di radiofarmaci atti a indagare in maniera più specifica diversi aspetti della biologia cellulare.

\section{Recenti applicazioni della diagnostica medico-nucleare in ambito oncologico}

La diagnostica per immagini riveste un ruolo chiave in Oncologia per la rilevazione precoce delle neoplasie, l'accurata stadiazione di malattia e la valutazione della risposta al trattamento. La TC e la RM forniscono importanti informazioni anatomiche, ma non offrono significative informazioni funzionali sulle lesioni rilevate; al contrario, l'imaging medico-nucleare "convenzionale" (planare e SPECT) e la PET possono fornire importanti informazioni funzionali aggiuntive, pur con una ridotta risoluzione spaziale rispetto alle metodiche radiologiche. Inoltre, grazie all'uso delle apparecchiature ibride SPECT/TC e PET/TC, tramite l'accurata co-registrazione di dati anatomici e funzionali, si ottengono informazioni complementari che in ambito oncologico si traducono in una maggiore sensibilità (superiore capacità di localizzare le lesioni) e una maggiore specificità (esclusione di falsi positivi dovuti al fisiologico accumulo del radiocomposto): ciò consente di definire correttamente il significato funzionale di lesioni indeterminate [2].

\section{Ruolo della SPECT/TC in Oncologia}

La SPECT/TC, tecnica ormai ampiamente disponibile, ha varie applicazioni in campo oncologico; riportiamo di seguito le più frequenti allo stato attuale.

\section{Imaging dei tumori neuroendocrini}

I tumori neuroendocrini (NET) sono un gruppo eterogeneo di neoplasie che include tumori benigni e maligni derivanti dalla cresta neurale quali il paraganglioma, il neuroblastoma, il 


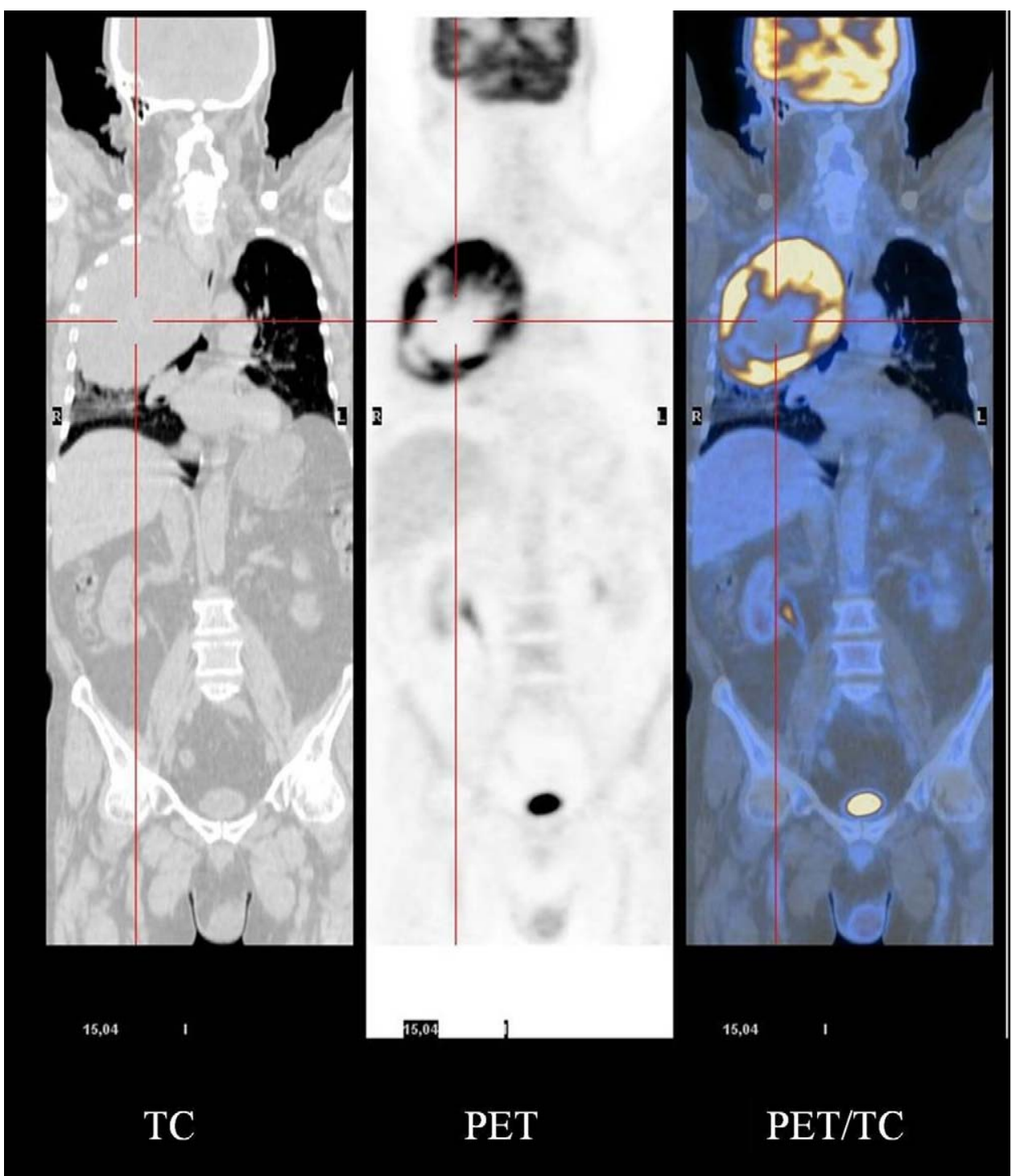

Figura 2 Paziente di 71 anni con voluminosa massa occupante gran parte dell'emitorace destro di riscontro TC. All'indagine PET/TC con FDG la massa appare caratterizzata da intensa e disomogenea attività metabolica per la presenza di estesa area necrotica centrale e irregolari margini ipercaptanti. La biopsia, effettuata sui margini della lesione, ha evidenziato un tessuto con caratteristiche di tipo sarcomatoso.

carcinoma midollare della tiroide e i tumori endocrini gastroentero-pancreatici (GEP). Spesso in pazienti con NET è richiesto un approccio diagnostico multimodale, dal momento che il tumore primitivo è a volte di piccole dimensioni e può non essere rilevato tramite la diagnostica radiologica convenzionale (TC o RM); pertanto, l'imaging funzionale mediconucleare permette una corretta localizzazione e stadiazione di queste neoplasie. La maggior parte delle neoplasie neuroendocrine esprime sulla superficie cellulare i recettori per la somatostatina (SSR), in particolare i sottotipi 2 e 5 . La scintigrafia con analoghi recettoriali della somatostatina (SRS), quali il pentetreotide marcato con indio-111, ha dimostrato un'elevata sensibilità nella localizzazione dei NET (80-100\%); fornisce, inoltre, utili informazioni prognostiche e aiuta a identificare quei pazienti che possono trarre beneficio dalla terapia con analoghi marcati della somatostatina [2-4].
La scintigrafia con MIBG marcata con iodio-123 o iodio131 , composto analogo delle catecolamine captato con meccanismo attivo e specifico dai tumori originanti dalla cresta neurale, mostra valori di sensibilità più elevati nelle neoplasie derivanti dalla midollare del surrene e dai paragangli (paragangliomi) e nei neuroblastomi. La MIBG radioiodata è captata anche in altri tipi di neoplasie neuroendocrine, nelle quali tuttavia la SRS mostra valori di sensibilità maggiori rispetto alla scintigrafia con MIBG (come nel caso dei GEP); in questi casi la scintigrafia con MIBG radioiodata può essere utilizzata quando altre metodiche diagnostiche non riescono a visualizzare i NETo in previsione di una terapia radiometabolica con MIBG marcata con iodio-131 [2-4].

Sia nel caso della SRS sia nella scintigrafia con MIBG l'accuratezza diagnostica della SPECT/TC è più elevata rispetto alla SPECT o alla TC da sole; l'imaging ibrido SPECT/TC nei NET permette infatti una migliore localizzazione anatomica delle 
lesioni, l'esclusione di lesioni in sedi di fisiologico accumulo del radiocomposto e l'identificazione di lesioni aggiuntive, apportando talvolta una modificazione nella gestione clinica del paziente [2-4].

\section{Imaging del carcinoma tiroideo differenziato}

Il carcinoma tiroideo differenziato (CDT) rappresenta la neoplasia endocrina più frequente e presenta generalmente una buona prognosi. La terapia convenzionale consiste nell'intervento chirurgico di tiroidectomia totale e nell'ablazione con iodio-131 del tessuto tiroideo residuo. Il follow-up del CDT viene effettuato tramite la misurazione dei valori di tireoglobulina, l'ecografia del collo e, se necessario, mediante la scintigrafia con iodio-131 (WBS). L'interpretazione della WBS può essere resa difficoltosa dalla mancanza di riferimenti anatomici e dal fisiologico accumulo del radioiodio nei residui di tessuto tiroideo, che possono simulare la presenza di metastasi.

L'imaging ibrido SPECT/TC incrementa sia la sensibilità sia la specificità della scintigrafia con radioiodio nei CDT, in particolare migliora l'accuratezza diagnostica identificando lesioni aggiuntive, permettendo una corretta localizzazione delle lesioni ed escludendo lesioni in sedi di fisiologico accumulo del radioiodio $[2,5]$.

\section{Ricerca del linfonodo sentinella}

La biopsia del linfonodo sentinella è un metodo affidabile di stadiazione in vari tumori, quali le neoplasie della mammella, i melanomi e i carcinomi squamosi della testa e del collo. La linfoscintigrafia rappresenta un prerequisito indispensabile per la localizzazione preoperatoria del linfonodo sentinella e per la successiva biopsia. La biopsia minimamente invasiva del linfonodo sentinella riduce la morbilità associata alla dissezione linfonodale, facilita un'appropriata scelta terapeutica e fornisce importanti informazioni prognostiche.

La linfoscintigrafia eseguita solo con tecnica planare può, in alcuni casi, non localizzare accuratamente la sede delle stazioni linfonodali di drenaggio e occasionalmente può non riconoscere linfonodi sentinella vicini alla lesione primitiva. Diversi studi hanno dimostrato la possibilità di migliorare sia la sensibilità sia la specificità della linfoscintigrafia attraverso le rilevazioni tomografiche SPECT/TC; la tecnica SPECT/TC, infatti, migliorerebbe l'accuratezza diagnostica permettendo una corretta localizzazione anatomica dei linfonodi e la visualizzazione di linfonodi non evidenziati dalla tecnica planare, in particolare vicino alla sede dell'iniezione $[2,6]$.

\section{Diagnostica dei secondarismi scheletrici}

Le lesioni scheletriche secondarie si presentano in circa il $30 \%$ dei pazienti oncologici. I tumori primitivi che metastatizzano più frequentemente a livello scheletrico sono $\mathrm{i}$ carcinomi originanti da mammella, prostata e polmone. La diagnosi precoce dei secondarismi scheletrici è indispensabile per una corretta stadiazione e per l'impostazione del piano di trattamento. La scintigrafia ossea tramite iniezione di difosfonati marcati con tecnezio-99m è una metodica ampiamente disponibile e permette la visualizzazione dell'intero scheletro con valori di sensibilità variabili dal $62 \%$ al $100 \%$ a seconda dei vari studi. Tuttavia, la specificità della metodica planare risulta bassa e in alcuni casi è possibile riscontrare reperti di non univoca interpretazione che possono essere meritevoli di ulteriore approfondimento diagnostico con metodiche radiologiche. L'imaging ibrido SPECT/TC consente di migliorare la sensibilità e la specificità della metodica e, in particolare, fornendo una corretta localizzazione anatomica delle lesioni, permette una corretta diagnosi di lesioni indeterminate all'imaging planare $[2,7,8]$.

\section{Ruolo della PET/TC in Oncologia}

Sono stati pubblicati numerosi studi sul ruolo della PET/TC in campo oncologico, soprattutto riguardo la diagnosi iniziale, la stadiazione, la ristadiazione e la valutazione della risposta al trattamento in varie neoplasie. La PET/TC può avere, inoltre, un ruolo importante nella pianificazione del trattamento radioterapico [1]. L'uso di FDG (radiofarmaco attualmente più utilizzato in PET) in ambito oncologico si basa sul fatto che le cellule tumorali, avendo un'attività metabolica maggiore rispetto alle cellule normali, accumulano più avidamente questo analogo marcato del glucosio. A differenza della TC, che richiede una modifica dimensionale in una determinata struttura anatomica per poter rilevare una neoplasia, la PET con FDG può evidenziare anche piccole quote di cellule tumorali all'interno di strutture anatomiche di normali dimensioni. Associando, mediante l'impiego di apparecchiature ibride PET/TC, l'elevata sensibilità della PET con FDG all'elevata risoluzione spaziale della TC si ottiene una maggiore accuratezza diagnostica nella visualizzazione dei processi neoplastici, superiore rispetto a quella offerta dalle singole metodiche PET e TC $[9,10]$.

Non tutte le neoplasie, tuttavia, sono ben visualizzabili con la PET con FDG; i tumori a lenta crescita, per esempio il carcinoma prostatico, a causa della loro bassa attività metabolica non mostrano solitamente significativo iperaccumulo di questo radiocomposto. Altri limiti del FDG sono la possibilità di falsi positivi dovuti a processi infiammatori e la ridotta sensibilità nello studio di strutture anatomiche caratterizzate da fisiologico accumulo del radiocomposto (per esempio, il cervello). Allo scopo di superare i limiti del FDG sono allo studio vari radiocomposti da usare nella PET, ciascuno dei quali permette di valutare un particolare processo metabolico (tabella 3) [11].

Qui di seguito riportiamo le principali applicazioni della $\mathrm{PET} / \mathrm{TC}$ in ambito oncologico allo stato attuale.

\section{Neoplasie polmonari}

La PET/TC con FDG è particolarmente utile nella caratterizzazione metabolica dei noduli polmonari solitari e nella stadiazione, ristadiazione e valutazione dell'efficacia del trattamento nei tumori polmonari non a piccole cellule (NSCLC). Possibili falsi positivi sono i processi infettivi e infiammatori; possibili cause di falsi negativi possono essere lesioni con dimensioni inferiori al limite di risoluzione della PET (circa $5 \mathrm{~mm}$ ) o neoplasie dotate di scarso accumulo di FDG (per esempio il carcinoma bronchiolo-alveolare o il carcinoide bronchiale) [11].

Nella valutazione del parametro T la PET/TC con FDG fornisce risultati superiori rispetto alla PET e alla TC da sole; in particolare, l'esatta correlazione tra l'iperaccumulo di FDG e i dati anatomici permette di valutare meglio 
l'infiltrazione della parete toracica, l'invasione mediastinica e la differenziazione tra tumore e atelettasia (quest'ultimo dato è molto importante nella pianificazione del trattamento radioterapico).

La PET/TC con FDG è una metodica eccellente nella stadiazione linfonodale in pazienti con NSCLC. Anche se il gold standard nella valutazione dei linfonodi mediastinici rimane la mediastinoscopia, la negatività del reperto PET, grazie all'elevata sensibilità della metodica, può consentire di escludere un interessamento linfonodale; al contrario, un reperto positivo impone una verifica mediante biopsia. Per la sua peculiarità di esame corporeo globale la PET/TC con FDG è utilissima nella valutazione delle metastasi a distanza, specialmente delle metastasi surrenaliche che sono spesso di difficile valutazione con la sola TC. La PET/TC con FDG è uno strumento molto sensibile nella valutazione di sospetta recidiva di malattia; tuttavia, la sua specificità si riduce se si effettua l'esame a breve distanza dalla terapia, a causa dell'accumulo di FDG nei tessuti irradiati e nelle flogosi postchirurgiche. La metodica può essere utile nel valutare la risposta alla chemioterapia e alla radioterapia nei tumori del polmone; una riduzione di accumulo di FDG dopo terapia, infatti, correla con la sopravvivenza a medio termine [11-16].

\section{Neoplasie esofagee}

Nel carcinoma esofageo la PET/TC con FDG ha un ruolo accertato nella stadiazione e nella valutazione della risposta alla terapia. Nello studio dei linfonodi locoregionali l'ecografia endoscopica sembra lo strumento più appropriato, mentre la PET/TC permette una valutazione delle metastasi a distanza sia in sede linfonodale sia negli altri organi. Nei pazienti con malattia localmente avanzata sottoposti a terapia neoadiuvante prima di una possibile resezione, la PET/TC sembra efficace nel selezionare quei pazienti che hanno avuto una risposta alla terapia, tale da meritare l'intervento chirurgico. Di fatto, la riduzione dell'accumulo di FDG a livello tumorale correla con l'efficacia della chemioterapia o della radioterapia $[11,17,18]$.

\section{Neoplasie del colon-retto}

La PET/TC con FDG non è indicata come esame di diagnosi iniziale delle neoplasie del colon-retto, considerata anche la possibile presenza di accumulo aspecifico del radiocomposto a livello intestinale. Per quanto riguarda la stadiazione, la $\mathrm{PET} / \mathrm{TC}$ ha un ruolo limitato nel valutare i parametri T e N, mentre mostra un'elevata sensibilità nel rilevare la presenza di metastasi a distanza, in particolare nel fegato. La PET/TC con FDG è però utilissima nella ristadiazione, nel valutare le recidive di malattia dopo la terapia, con un impatto significativo sulla successiva gestione clinica del paziente. In particolare, la metodica è utile nel confermare e localizzare siti di ripresa di malattia in pazienti con incremento dei valori di antigene carcinoembrionico (CEA) quando le indagini radiologiche (ecografia e TC) risultano negative $[11,19,20]$.

\section{Melanoma}

Il melanoma è uno dei tumori che accumula più avidamente FDG. La PET/TC è molto utile nel valutare le metastasi a distanza (eccetto localizzazioni secondarie di piccole dimensioni e metastasi cerebrali), mentre è meno sensibile nel valutare metastasi ai linfonodi locoregionali; in particolare, non è in grado di valutare la presenza di micrometastasi a livello del linfonodo sentinella [11,21,22].

\section{Linfoma}

Allo stato attuale la PET/TC con FDG è la metodica più adeguata nella valutazione dell'estensione di malattia e nel follow-up in pazienti con linfoma e ha mostrato, inoltre, un'elevata accuratezza diagnostica nella ristadiazione dopo il trattamento iniziale. La PET con FDG permette altresì di predire in maniera precoce la risposta alla terapia e di valutare masse residue dopo chemioterapia o radioterapia [11,23,24].

\section{Neoplasie della testa e del collo}

Nei tumori della testa e del collo, in particolare i carcinomi squamocellulari e i tumori derivanti dalle ghiandole salivari, la PET/TC con FDG non svolge un ruolo significativo nella caratterizzazione del tumore primitivo. In questo ambito, le informazioni fornite da TC con mezzo di contrasto e RM risultano superiori. Il ruolo primario della PET/TC con FDG nei tumori della testa e del collo attiene invece alla valutazione di metastasi linfonodali locoregionali e di metastasi a distanza. La PET/TC con FDG ha inoltre un ruolo importante nel follow-up, nella rilevazione di eventuali recidive e nella valutazione della risposta alla chemioterapia e alla radioterapia $[11,25,26]$.

\section{Neoplasie mammarie}

La PET/TC con FDG ha un ruolo limitato nella diagnosi iniziale del carcinoma mammario, tuttavia ha un ruolo importante nel rilevare la malattia locoregionale (incluso l'interessamento linfonodale) e a distanza, nel monitorare la risposta alla terapia e nell'evidenziare recidive di malattia. La metodica è inoltre importante nella pianificazione del trattamento radioterapico $[11,12,27]$.

\section{Neoplasie tiroidee differenziate}

Nelle neoplasie tiroidee differenziate la PET/TC con FDG ha un ruolo riconosciuto nella ristadiazione, in particolare nella valutazione di pazienti con sospetta recidiva di malattia, elevati valori di tireoglobulina e WBS con iodio-131 negativo $[11,12,28]$.

\section{Neoplasie ginecologiche}

Utero e ovaie (in fase premenopausale) possono essere occasionalmente sede di fisiologico accumulo di FDG, pertanto la $\mathrm{PET} / \mathrm{TC}$ con FDG non risulta indicata nella valutazione del parametro $T$ in queste neoplasie. Nelle neoplasie uterine la $\mathrm{PET} / \mathrm{TC}$ con FDG può essere tuttavia utilizzata nella stadiazione (in aggiunta all'imaging radiologico convenzionale), nella ristadiazione e nella valutazione della risposta alla terapia. Per quanto riguarda il carcinoma ovarico si ritiene che la PET/TC con FDG possa essere la metodica di scelta nella ristadiazione di tale neoplasia, soprattutto nella ricerca di eventuale recidive in pazienti con aumento di $\mathrm{Ca}-125$ $[11,12,29]$.

\section{Altre neoplasie}

Nelle neoplasie delle vie biliari la PET/TC con FDG sembra avere un ruolo promettente nella stadiazione e ristadiazione [30]. 
Nella stadiazione delle neoplasie pancreatiche la PET/TC potrebbe fornire informazioni aggiuntive rispetto alla TC con mezzo di contrasto e all'ecografia endoscopica, che rimangono metodiche di prima istanza [31].

Nei tumori a cellule germinali e nei tumori stromali gastrointestinali (GIST) la PET/TC con FDG può avere un ruolo nella ristadiazione e nella valutazione della risposta alla terapia $[11,12,32]$.

L'accumulo di FDG è generalmente variabile nei tumori dell'apparato urinario, nei sarcomi e nei tumori neuroendocrini [11]. I tumori del rene, dell'uretere e della vescica sono difficili da studiare mediante la PET/TC con FDG a causa del fisiologico accumulo del radiocomposto a livello urinario; tuttavia, in questi tumori le lesioni secondarie mostrano spesso un accumulo di FDG superiore alla lesione primitiva, indice di una maggiore aggressività delle lesioni secondarie [11]. I sarcomi rappresentano un gruppo eterogeneo di neoplasie derivanti dai tessuti molli e dallo scheletro e mostrano un variabile accumulo di FDG [11]. La PET/TC con FDG ha valore limitato nello studio delle neoplasie neuroendocrine e solo neoplasie scarsamente differenziate o con alti indici proliferativi presentano un iperaccumulo di FDG; la PET con traccianti basati sulle caratteristiche metaboliche (come la fluoro-18-DOPA) e recettoriali (come gli analoghi della somatostatina marcati con gallio-68) dei tumori neuroendocrini ha mostrato risultati promettenti in questo tipo di neoplasie [3].

Gli adenocarcinomi ricchi di mucina (come gli adenocarcinomi gastrici), i tumori ben differenziati del fegato, il carcinoma bronchiolo-alveolare, il tumore della prostata e i tumori cerebrali primitivi (eccetto il glioblastoma multiforme) mostrano ridotto accumulo di FDG [11].

I derivati della colina marcati con carbonio-11 o fluoro-18 sono radiofarmaci utilissimi nello studio del tumore della prostata, in particolare nella stadiazione preintervento e nella valutazione di recidive in pazienti con incremento dei valori di antigene prostatico specifico; al contrario, la PET con colina non è utile per distinguere tra il tumore della prostata e l'ipertrofia prostatica [12].

Nello studio dei tumori cerebrali primitivi la RM rimane il gold standard, tuttavia la metodica presenta una bassa specificità nel differenziare tra recidive di malattia e alterazioni post-terapia (chirurgia, chemioterapia o radioterapia). Riguardo alle neoplasie cerebrali primitive, la PET con FDG permette di valutare la trasformazione in senso anaplastico della neoplasia, ma presenta un'accuratezza diagnostica inferiore rispetto alla PET con traccianti aminoacidici (come la metionina marcata con carbonio-11) nello studio dei tumori cerebrali a basso grado. In particolare, la PET con traccianti aminoacidici costituisce uno strumento accurato per la diagnosi differenziale tra recidiva di malattia e modifiche anatomiche post-terapia [33].

\section{Conflitto di interesse}

Gli autori dichiarano di essere esenti da conflitto di interessi.

\section{Bibliografia}

[1] Maurer AH. Combined imaging modalities: PET/CT and SPECT/ CT. Health Phys 2008;95(5):571-6.
[2] Chowdhury FU, Scarsbrook AF. The role of hybrid SPECT-CT in oncology: current and emerging clinical applications. Clin Radiol 2008;63(3):241-51.

[3] Rufini V, Calcagni ML, Baum RP. Imaging of neuroendocrine tumors. Semin Nucl Med 2006;36(3):228-47.

[4] Schillaci O. Functional-anatomical image fusion in neuroendocrine tumors. Cancer Biother Radiopharm 2004;19(1):129-34.

[5] Tharp K, Israel O, Hausmann J, Bettman L, Martin WH, Daitzchman M, et al. Impact of 131I-SPECT/CT images obtained with an integrated system in the follow-up of patients with thyroid carcinoma. Eur J Nucl Med Mol Imaging 2004;31(10):1435-42.

[6] Even-Sapir E, Lerman H, Lievshitz G, Khafif A, Fliss DM, Schwartz $A$, et al. Lymphoscintigraphy for sentinel node mapping using a hybrid SPECT/CT system. J Nucl Med 2003;44(9):1413-20.

[7] Even-Sapir E. Imaging of malignant bone involvement by morphologic, scintigraphic, and hybrid modalities. J Nucl Med 2005;46(8):1356-67.

[8] Römer W, Nömayr A, Uder M, Bautz W, Kuwert T. SPECT-guided CT for evaluating foci of increased bone metabolism classified as indeterminate on SPECT in cancer patients. J Nucl Med 2006;47(7):1102-6.

[9] Townsend DW. Positron emission tomography/computed tomography. Semin Nucl Med 2008;38(3):152-66.

[10] Mawlawi O, Townsend DW. Multimodality imaging: an update on PET/CT technology. Eur J Nucl Med Mol Imaging 2009; 36(Suppl 1):S15-29.

[11] Mittra E, Quon A. Positron emission tomography/computed tomography: the current technology and applications. Radiol Clin North Am 2009;47(1):147-60.

[12] Von Schulthess GK, Hany TF. Imaging and PET-PET/CT imaging. J Radiol 2008;89(3 Pt 2):438-47.

[13] Lardinois D, Weder W, Hany TF, Kamel EM, Korom S, Seifert B, et al. Staging of non-small-cell lung cancer with integrated positron-emission tomography and computed tomography. $\mathrm{N}$ Engl J Med 2003;348(25):2500-7.

[14] Keidar Z, Haim N, Guralnik L, et al. PET/CT using 18F-FDG in suspected lung cancer recurrence: diagnostic value and impact on patient management. J Nucl Med 2004;45(10):1640-6.

[15] Antoch G, Stattaus J, Nemat AT, Marnitz S, Beyer T, Kuehl H, et al. Non-small cell lung cancer: dual-modality PET/CT in preoperative staging. Radiology 2003;229(2):526-33.

[16] Ciernik IF, Dizendorf E, Baumert BG, Reiner B, Burger C, Davis $\mathrm{JB}$, et al. Radiation treatment planning with an integrated positron emission and computer tomography (PET/CT): a feasibility study. Int J Radiat Oncol Biol Phys 2003;57(3):853-63.

[17] Wallace MB, Nietert PJ, Earle C, Krasna MJ, Hawes RH, Hoffman $\mathrm{BJ}$, et al. An analysis of multiple staging management strategies for carcinoma of the esophagus: computed tomography, endoscopic ultrasound, positron emission tomography, and thoracoscopy/laparoscopy. Ann Thorac Surg 2002;74(4):1026-32.

[18] Brücher BL, Weber W, Bauer M, Fink U, Avril N, Stein HJ, et al. Neoadjuvant therapy of esophageal squamous cell carcinoma: response evaluation by positron emission tomography. Ann Surg 2001;233(3):300-9.

[19] Esteves FP, Schuster DM, Halkar RK. Gastrointestinal tract malignancies and positron emission tomography: an overview. Semin Nucl Med 2006;36(2):169-81.

[20] Pelosi E, Deandreis D. The role of $18 \mathrm{~F}$-fluoro-deoxy-glucose positron emission tomography (FDG-PET) in the management of patients with colorectal cancer. Eur J Surg Oncol 2007; $33(1): 1-6$.

[21] Swetter SM, Carroll LA, Johnson DL, Segall GM. Positron emission tomography is superior to computed tomography for metastatic detection in melanoma patients. Ann Surg Oncol 2002;9(7):646-53.

[22] Wagner JD, Schauwecker D, Davidson D, Logan T, Coleman 3rd JJ, Hutchins G, et al. Inefficacy of F-18 fluorodeoxy-D-glucosepositron emission tomography scans for initial evaluation 
in early-stage cutaneous melanoma. Cancer 2005;104(3): 570-9.

[23] Pelosi E, Pregno P, Penna D, Deandreis D, Chiappella A, Limerutti $\mathrm{G}$, et al. Role of whole-body [18F] fluorodeoxyglucose positron emission tomography/computed tomography (FDG-PET/CT) and conventional techniques in the staging of patients with Hodgkin and aggressive non Hodgkin lymphoma. Radiol Med 2008;113(4):578-90.

[24] Altamirano J, Esparza JR, de la Garza Salazar J, Calvo PS, Vera SR, Chalapud Revelo JR, et al. Staging, response to therapy, and restaging of lymphomas with 18F-FDG PET. Arch Med Res 2008;39(1):69-77.

[25] Yamazaki Y, Saitoh M, Notani K, Tei K, Totsuka Y, Takinami S, et al. Assessment of cervical lymph node metastases using FDGPET in patients with head and neck cancer. Ann Nucl Med 2008;22(3):177-84.

[26] Tan A, Adelstein DJ, Rybicki LA, Saxton JP, Esclamado RM, Wood BG, et al. Ability of positron emission tomography to detect residual neck node disease in patients with head and neck squamous cell carcinoma after definitive chemoradiotherapy. Arch Otolaryngol Head Neck Surg 2007;133(5): 435-40.

[27] Zangheri B, Messa C, Picchio M, Gianolli L, Landoni C, Fazio F. PET/CT and breast cancer. Eur J Nucl Med Mol Imaging 2004; 31(Suppl 1):S135-42.
[28] Shammas A, Degirmenci B, Mountz JM, McCook BM, Branstetter $B$, Bencherif $B$, et al. 18F-FDG PET/CT in patients with suspected recurrent or metastatic welldifferentiated thyroid cancer. J Nucl Med 2007;48(2):221-6.

[29] Grisaru D, Almog B, Levine C, Metser U, Fishman A, Lerman H, et al. The diagnostic accuracy of $18 \mathrm{~F}$-fluorodeoxyglucose PET/ $\mathrm{CT}$ in patients with gynaecological malignancies. Gynecol Oncol 2004;94(3):680-4.

[30] Petrowsky H, Wildbrett P, Husarik DB, Hany TF, Tam S, Jochum $\mathrm{W}$, et al. Impact of integrated positron emission tomography and computed tomography on staging and management of gallbladder cancer and cholangiocarcinoma. J Hepatol 2006;45(1): 43-50.

[31] Heinrich S, Goerres GW, Schäfer M, Sagmeister M, Bauerfeind P, Pestalozzi BC, et al. Positron emission tomography/computed tomography influences on the management of resectable pancreatic cancer and its cost-effectiveness. Ann Surg 2005; 242(2):235-43.

[32] Goerres GW, Stupp R, Barghouth G, Hany TF, Pestalozzi B, Dizendorf $\mathrm{E}$, et al. The value of PET, CT and in-line PET/CT in patients with gastrointestinal stromal tumours: long-term outcome of treatment with imatinib mesylate. Eur J Nucl Med Mol Imaging 2005;32(2):153-62.

[33] Chen W, Silverman DH. Advances in evaluation of primary brain tumors. Semin Nucl Med 2008;38(4):240-50. 